Article

\title{
Association between Total Sugar Intake and Metabolic Syndrome in Middle-Aged Korean Men and Women
}

\author{
Eun Ha Seo ${ }^{1}$, Hyesook Kim ${ }^{2, * \mathbb{D}}$ and Oran Kwon ${ }^{1,2, * \mathbb{D}}$ \\ 1 Department of Clinical Nutrition Science, The Graduate School of Clinical Health Sciences, \\ Ewha Womans University, 52, Ewhayeodae-gil, Seodaemun-gu, Seoul 03760, Korea \\ 2 Department of Nutritional Science and Food Management, Ewha Womans University, 52, Ewhayeodae-gil, \\ Seodaemun-gu, Seoul 03760, Korea \\ * Correspondence: khs7882@hanmail.net (H.K.); orank@ewha.ac.kr (O.K.); Tel./Fax: +82-2-3277-6860 (H.K. \& O.K.)
}

Received: 29 July 2019; Accepted: 28 August 2019; Published: 1 September 2019

\begin{abstract}
There is increasing evidence emerging that suggests high sugar intake may adversely increase the incidence of chronic diseases. However, there are only a few related studies in Korea. Based on the current Dietary Reference Intakes for Koreans, this study examined whether total sugar intake above $20 \%$ of the total energy was a risk factor for metabolic syndrome in middle-aged Korean adults. This cross-sectional study involved 7005 adults (3751 men and 3254 women) aged 40-69 years, who participated in the Korean Genome and Epidemiology Study (KoGES), a large community-based cohort study. Daily total sugar intake was estimated using a validated food frequency questionnaire. About $9 \%$ and $16 \%$ of the men and women, respectively, derived $>20 \%$ of energy intake from total sugar. The males in this category had a significantly higher odds of obesity defined as having a $\mathrm{BMI} \geq 25$ ( $\mathrm{OR}=1.491,95 \% \mathrm{CI}=1.162-1.914)$, low HDL-cholesterol $(\mathrm{OR}=1.313$, $95 \% \mathrm{CI}=1.038-1.660)$, and metabolic syndrome ( $\mathrm{OR}=1.332,95 \% \mathrm{CI}=1.038-1.709)$ than those who received a lower proportion of energy intake from total sugar. These results suggest that high $(>20 \%)$ energy intake from total sugar may be associated with an increased risk of metabolic syndrome in middle-aged Korean men.
\end{abstract}

Keywords: total sugar; chronic disease; Korean Genome and Epidemiology Study (KoGES)

\section{Introduction}

Unhealthy eating patterns and a growing prevalence of chronic diseases, including diabetes and obesity, are a global concern. Healthy food choice is closely linked with the quality of life and healthy life expectancy, as well as the burden of healthcare expenditure. Consequently, among researchers, the public, and policymakers alike, there is a strong desire to identify the risk factors of diet-related chronic diseases [1]. In accord with this pursuit, there is a growing interest in the role of dietary patterns in the etiology of chronic diseases. While there have been many studies on fat intake associated with chronic diseases, sugar consumption has become a major concern in relation to the development of cardiovascular and metabolic diseases.

Biochemically, sugars consist of monosaccharides (glucose, fructose, galactose) and disaccharides (sucrose, maltose, lactose), excluding oligosaccharides [2]. Sugars occur naturally in some food, such as fruits and vegetables [3], in co-existence with other micronutrients and, therefore, have nutritional value [4]. By contrast, added sugars, which include refined sugars, honey, syrups, and other caloric sweeteners added to food or meals during processing or at home [2], provide no nutritional benefits [4]. Sugar-sweetened beverages (SSB) include sodas, sweetened juices, energy drinks, teas, and coffee that have been sweetened with added sugar $[2,5]$. 
Excessive intake of energy-dense sugary foods and beverage is linked to increased calorie intake and progressive body weight gain. Notably, high fructose consumption has been proposed as casually linked to obesity, diabetes, and hypertension, which besides, hypertriglyceridemia, low-HDL levels, and insulin resistance, are risk factors for metabolic syndrome [6]. Although the rationale that dietary sugar (particularly fructose) might cause or contribute to the disease process is theoretically based, it has raised debate around the amounts and thresholds of sugar consumption.

In 2015, the World Health Organization (WHO) recommended that countries committed to reducing the burden of non-communicable diseases should limit the intake of "free sugar" (added sugar, as well as sugars naturally present in fruit juice and fruit concentrates) in children and adults to less than $10 \%$ of the total energy intake (TEI) and, ideally, to below $5 \%$ for additional health benefits [7]. In the United States (US), the 2010 Dietary Guidelines Advisory Committee recommended that solid fat and added sugars should account for no more than 5-15\% of the TEI [8], and in 2007, the United Kingdom (UK) proposed an average upper limit of 11\% TEI from non-milk extrinsic sugars [9]. The 2015 Dietary Reference Intakes for Koreans (KDRI) direct a daily average sugar intake of $10-20 \%$ of the TEI (100 g based on $2000 \mathrm{kcal}$ ), and an added sugar (e.g., sugar, liquid fructose, starch syrup, molasses, honey, syrup, concentrated fruit juice) intake of within $10 \%$ of the TEI ( $50 \mathrm{~g}$ per $2000 \mathrm{kcal}$ ) [10]. These guidelines were justified by the fact that, when the percentage of TEI from total sugar is below $20 \%$, as the total sugar intake increases, the intake of major nutrients also increases [11]. Meanwhile, for individuals with above $20 \%$ TEI from sugar, as the total sugar intake increases, the intake of some nutrients, such as protein, fat, sodium, and niacin, decreases [11] and the risk of metabolic syndrome increases [12].

According to the data estimating the consumption of sugars in various countries, daily mean total sugar intake for adults were $118.5 \mathrm{~g}$ in the US from 2009 to 2010, $95.1 \mathrm{~g}$ in the UK from 2008 to 2012, $104.8 \mathrm{~g}$ in Australia from 2011 to 2012, and $87.8 \mathrm{~g}$ in Sweden from 2010 to 2011 [13]. In Korea, daily mean total sugar intake from 2008 to 2011 was estimated as $61.4 \mathrm{~g}$ (percentage of energy from total sugar was 12.8\%) according to the Korean National Health and Nutrition Examination Survey (KNHANES) [11]. When compared with western countries, total sugar intake among Koreans are relatively lower. However, the average daily total sugar intake of Koreans have gradually increased from $69.9 \mathrm{~g}$ in 2010 to $76.9 \mathrm{~g}$ in 2015, and when men and women were compared, the men's intake was relatively higher (81.4 $\mathrm{g}$ in men and $72.4 \mathrm{~g}$ in women) [14].

Although the sugar intake of Koreans is not higher than that of western countries, over the past decades, the frequency of eating out and the intake of high sugar levels and processed food which are the characteristics of a Western diet, have been increasing $[15,16]$ and are coincident with an increased prevalence of metabolic syndrome [17]. Around $27 \%$ of the entire Korean population are reported to have metabolic syndrome (30\% in men and about $25 \%$ in women) [18]. Hence, careful attention should be paid to sugar intake in Korea. Moreover, the difference in the prevalence of metabolic syndrome between sexes [19] highlights the importance of examining the relationship between total sugar intake and the risk of metabolic syndrome in adult men and women, especially those at risk of chronic diseases; yet there is a lack of related research in Korea. Therefore, this study examined whether a total sugar intake above $20 \%$ of the TEI (based on the current KDRI recommended level for total sugar intake) is a risk factor for metabolic syndrome in middle-aged Korean adult men and women who participated in the 2001-2002 Korean Genome and Epidemiology Study (KoGES).

\section{Materials and Methods}

\subsection{Study Population}

This study performed a cross-sectional analysis of the 2001-2002 data provided by the KoGES, a prospective, community-based cohort study that started in 2001 and since then, conducts biennial follow-up studies. The KoGES analyzes the effect of lifestyle, intake, and environment on the incidence of chronic disease in participants aged 40-69 years living in community residences. Two South Korean 
communities were selected, one from Ansung, representing a rural community, and the other from Ansan, representing an urban community. Detailed information on the study procedure was described in a previous report [20].

Among 10,030 participants, those diagnosed with myocardial infarction, stroke, coronary artery disease, congestive heart failure, and history of cancer were excluded $(n=436)$. The following participants were also excluded due to an incomplete food frequency questionnaire (FFQ) $(n=691)$, unreliable energy intake of $\leq 500 \mathrm{kcal}$ or $>5000 \mathrm{kcal}(n=76)$, missing data regarding metabolic syndrome-related indicators $(n=243)$, and missing covariates, such as menopausal status $(n=1,579)$. Finally, a total of 7005 subjects (3751 men and 3254 women) were included for this cross-sectional analysis.

\subsection{General Characteristics}

Individual questionnaires, including lifestyle and disease history questions, were filled out by all participants. Residential location was divided as Ansan and Ansung. Three levels of education (elementary school, middle school/high school, and college/graduate school), alcohol consumption (non-drinker, ex-drinker, current drinker), and smoking behavior (non-smoker, ex-smoker, current smoker) were formed. Physical activity was defined as none or more than $30 \mathrm{~min} /$ day. Menopausal status was defined as "yes" or "no", depending on whether menstruation had occurred for 12 months or more.

\subsection{Measurement of Anthropometry and Metabolic Parameters}

Anthropometry and metabolic parameters were measured by trained medical staff, with subjects wearing light clothes. Body mass index (BMI) was calculated as weight $(\mathrm{kg})$ divided by the square of the height $\left(\mathrm{m}^{2}\right)$. Blood pressure measurement was conducted at least twice under comfortable conditions. Blood samples obtained from the participants who fasted for $8 \mathrm{~h}$ were analyzed for biochemical markers, such as HDL-cholesterol, triglycerides, fasting blood glucose, insulin, C-reactive protein, and glycosylated hemoglobin. The homeostatic model assessment of insulin resistance ([fasting insulin $\times$ fasting glucose]/405) [21], LDL-cholesterol (total cholesterol—HDL-cholesterol—[triglycerides]/5]) [21], and atherogenic index ([total cholesterol-HDL-cholesterol]/HDL-cholesterol) [22] were calculated as indicated.

\subsection{Definitions of Metabolic Syndrome}

Metabolic syndrome was diagnosed using two approaches: The Adult Treatment Panel III of the National Cholesterol Education Program's (NCEP ATP III) standard diagnostic criteria [23], and the Korean Society for the Study of Obesity guidelines on waist circumference (WC) for defining abdominal obesity [24]. Metabolic syndrome was defined as having three or more of the following:

- WC: Male $>90 \mathrm{~cm}$, female $>85 \mathrm{~cm}$

- Systolic blood pressure $\geq 130 \mathrm{mmHg}$, diastolic blood pressure $\geq 85 \mathrm{mmHg}$

- Fasting blood glucose $\geq 100 \mathrm{mg} / \mathrm{dL}$

- Triglycerides $\geq 150 \mathrm{mg} / \mathrm{dL}$

- HDL-cholesterol: Male $<40 \mathrm{mg} / \mathrm{dL}$, female $<50 \mathrm{mg} / \mathrm{dL}$

\subsection{Dietary Assessment}

Dietary intake was assessed using a validated 103-item FFQ. All study procedures were conducted by trained interviewers. Participants were asked to report the usual frequency and portion size of each food consumed during the past year. The answer for frequency had nine options for each food: "never/almost," "once/month," "2-3 times/month," "1-2 times/week," "3-4 times/week," "5-6 times/week," "once/day," "2 times/day," and " $\geq 3$ times/day." The answer for portion size had three options for each food: "small (1/2 serving/day)," "standard (1 serving/day)," and "large ( $\geq 2$ servings/day)." The portion size of each 
food was determined based on the KoGES FFQ guideline. For analysis, food consumption was converted to weekly frequencies and then multiplied by the reported portion sizes.

Dietary nutrient intake data were compared using the estimated energy requirement and the estimated average requirement of the 2015 KDRI [10].

\section{Estimation of Total Sugar Intake}

Daily total sugar intake was estimated from the total sugar database constructed by the Korea Food and Drug Administration (KFDA) [25] for the foods included in the FFQ recipe. The percentage of energy from sugar intake was calculated as daily total sugar intake $\times 4 \mathrm{kcal} /$ energy intake $\times 100$.

\subsection{Statistical Analysis}

Data were expressed as mean \pm standard deviation for continuous variables and number $(n, \%)$ for categorical variables. To identify the significance of the difference in means and distribution of general characteristics and dietary intakes between the two groups ( $\leq 20 \%$ TEI from total sugar vs. $>20 \%$ TEI from total sugar), the Student's t-test (for continuous variables: Age, BMI) and the chi-square test and Fisher's exact test (categorical variables: residential location, educational level, alcohol consumption, smoking behavior, physical activity, menopausal status) were used.

Differences in metabolic parameters between the two groups were evaluated using the general linear model (GLM) test after adjusting for potential confounders. The potential confounders included the following variables: Age, energy intake, residential location, educational level, alcohol consumption, smoking behavior, physical activity, and menopausal status.

Multiple logistic regression analysis was performed to estimate the odds ratios (OR) and 95\% confidence intervals (CI) for cardiometabolic biomarkers and metabolic syndrome, depending on TEI from total sugar ( $\leq 20 \%$ vs. $>20 \%$ ). Potential confounders were included in the GLM and multiple logistic regression analysis as covariates. All statistical analyses were performed using SAS software version 9.4. Statistical significance was considered when $p<0.05$.

\section{Results}

\subsection{General Characteristics of the Subjects According to the Percentage of Energy from Total Sugar Intake}

The general characteristics of the two groups $(\leq 20 \%$ TEI from total sugar vs. $>20 \%$ TEI from total sugar) are displayed in Table 1. Mean total sugar intakes and mean percentage TEI from total sugar of men and women were $66.5 \pm 40.6$ and $68.0 \pm 45.7 \mathrm{~g} /$ day and $12.8 \pm 5.4 \%$ and $14.4 \pm 6.7 \% /$ day, respectively (data not shown). About $9 \%$ of the men and $16 \%$ of the women derived $>20 \%$ TEI from total sugar. The males in this category had a lower proportion of current drinkers, a higher proportion of current smokers, and were significantly older than the males who received $\leq 20 \%$ TEI from total sugar. Regarding residential location, the proportions of men and women who had $>20 \%$ TEI from total sugar were significantly higher in Ansung than Ansan $(p<0.0001)$. In women, there was no difference in general characteristics between the two groups $(\leq 20 \%$ TEI from total sugar vs. $>20 \%$ TEI from total sugar), except for residential location. 
Table 1. General characteristics of participants according to the percentage of energy from total sugar intake ${ }^{1}$.

\begin{tabular}{|c|c|c|c|c|c|c|}
\hline \multirow[b]{2}{*}{ Characteristic } & \multicolumn{3}{|c|}{ Men $(n=3751)$} & \multicolumn{2}{|c|}{ Women $(n=3254)$} & \multirow[b]{2}{*}{$p$-Value ${ }^{2}$} \\
\hline & $\begin{array}{c}\% \text { Energy from } \\
\text { Total Sugar } \leq 20 \\
(n=3404)\end{array}$ & $\begin{array}{c}\% \text { Energy from } \\
\text { Total Sugar }>20 \\
(n=347)\end{array}$ & $p$-Value ${ }^{2}$ & $\begin{array}{c}\% \text { Energy from } \\
\text { Total Sugar } \leq 20 \\
(n=2734)\end{array}$ & $\begin{array}{c}\text { \%Energy from } \\
\text { Total Sugar }>20 \\
(n=520)\end{array}$ & \\
\hline Age (years) & $51.0 \pm 8.6$ & $52.4 \pm 8.9$ & 0.0047 & $53.3 \pm 9.1$ & $53.5 \pm 9.0$ & 0.6515 \\
\hline BMI $\left(\mathrm{kg} / \mathrm{m}^{2}\right)$ & $24.2 \pm 2.9$ & $24.4 \pm 3.1$ & 0.4813 & $24.9 \pm 3.3$ & $25.1 \pm 3.2$ & 0.2371 \\
\hline Residential location & & & $<0.0001$ & & & $<0.0001$ \\
\hline Ansung & $1251(36.8)$ & $214(61.7)$ & & $1351(49.4)$ & $338(65.0)$ & \\
\hline Ansan & 2153 (63.3) & 133 (38.3) & & 1383 (50.6) & $182(35.0)$ & \\
\hline Educational level & & & 0.1448 & & & 0.5457 \\
\hline Below elementary school & $635(18.7)$ & $76(21.9)$ & & $1269(46.4)$ & $244(46.9)$ & \\
\hline Middle school/high school & $2025(59.5)$ & $188(54.2)$ & & $1294(47.3)$ & $250(48.1)$ & \\
\hline College/graduate school & $744(21.9)$ & $83(23.9)$ & & $171(6.3)$ & $26(5.0)$ & \\
\hline Alcohol consumption & & & 0.0209 & & & 0.1718 \\
\hline Non-drinker & $600(17.6)$ & $81(23.3)$ & & $1989(72.8)$ & $368(70.8)$ & \\
\hline Ex-drinker & $316(9.3)$ & $35(10.1)$ & & $75(2.7)$ & $22(4.2)$ & \\
\hline Current drinker & $2488(73.1)$ & $231(66.6)$ & & $670(24.5)$ & $130(25.0)$ & \\
\hline Smoking behavior & & & 0.0126 & & & 0.5062 \\
\hline Non-smoker & $661(19.4)$ & $72(20.8)$ & & $2604(95.3)$ & $501(96.4)$ & \\
\hline Ex-smoker & $1074(31.6)$ & 83 (23.9) & & $35(1.3)$ & $6(1.2)$ & \\
\hline Current smoker & $1669(49.0)$ & $192(55.3)$ & & $95(3.5)$ & $13(2.5)$ & \\
\hline Physical activity ${ }^{3}$ & & & 0.2561 & & & 0.1902 \\
\hline No & $112(3.3)$ & $16(4.6)$ & & $13(0.5)$ & $5(1.0)$ & \\
\hline Yes & 3292 (96.7) & $331(95.4)$ & & $2721(99.5)$ & $515(99.0)$ & \\
\hline Menopausal status & & & & & & 0.6007 \\
\hline No & & & & 1104 (40.4) & $203(39.0)$ & \\
\hline Yes & & & & 1630 (59.6) & $317(61.0)$ & \\
\hline
\end{tabular}

${ }^{1}$ Data are expressed as mean \pm standard deviation or $n(\%) .{ }^{2}$ Student's $t$-test for continuous variables, and chi-square or Fisher's exact test for categorical variables were used to analyze $p$-value. ${ }^{3}$ Physical activity was defined as "yes" or "no", depending on whether or not exercise was performed for $\geq 30 \mathrm{~min} /$ day. BM1, body mass index.

\subsection{Daily Nutrient Intake According to the Percentage of Energy from Total Sugar Intake}

In men and women, daily intakes of all macronutrients, including energy, were significantly higher in the group with $>20 \%$ TEI from total sugar than the group with $\leq 20 \%$ TEI from total sugar (Table 2 ). Vitamin and mineral intakes showed the same trend (data not shown). When comparing the two groups as contribution percentage in the energy intake (i.e., not the absolute intake of macronutrients), the percentage of energy intake from carbohydrate in men, and the percentage of energy intake from fat and carbohydrate in women were significantly higher in the group with $>20 \%$ TEI from total sugar.

Table 2. Daily intakes of energy and nutrient in the subjects according to the percentage of energy from total sugar intake ${ }^{1}$.

\begin{tabular}{|c|c|c|c|c|c|c|}
\hline \multirow[b]{2}{*}{ Intake Parameter } & \multicolumn{3}{|c|}{ Men $(n=3751)$} & \multicolumn{3}{|c|}{ Women $(n=3254)$} \\
\hline & $\begin{array}{c}\text { \%Energy from } \\
\text { Total Sugar } \leq 20 \\
(n=3404)\end{array}$ & $\begin{array}{c}\text { \%Energy from } \\
\text { Total Sugar }>20 \\
\quad(n=347)\end{array}$ & $p$-Value ${ }^{2}$ & $\begin{array}{l}\text { \%Energy from } \\
\text { Total Sugar } \leq 20 \\
\quad(n=2734)\end{array}$ & $\begin{array}{c}\text { \%Energy from } \\
\text { Total Sugar }>20 \\
\quad(n=520)\end{array}$ & $p$-Value ${ }^{2}$ \\
\hline Energy (kcal/day) & $1982.5 \pm 550.2$ & $2303.9 \pm 752.0$ & $<0.0001$ & $1819.7 \pm 603.6$ & $2071.9 \pm 712.6$ & $<0.0001$ \\
\hline Protein (g/day) & $68.6 \pm 25.0$ & $78.1 \pm 29.1$ & $<0.0001$ & $60.6 \pm 24.6$ & $69.0 \pm 27.9$ & $<0.0001$ \\
\hline Fat (g/day) & $35.3 \pm 18.1$ & $40.9 \pm 20.3$ & $<0.0001$ & $27.3 \pm 16.9$ & $32.3 \pm 17.3$ & $<0.0001$ \\
\hline Carbohydrate (g/day) & $341.3 \pm 88.9$ & $408.6 \pm 136.2$ & $<0.0001$ & $326.9 \pm 105.9$ & $380.0 \pm 133.0$ & $<0.0001$ \\
\hline$\%$ Energy from protein & $13.7 \pm 2.3$ & $13.6 \pm 2.3$ & 0.4019 & $13.2 \pm 2.3$ & $13.3 \pm 2.6$ & 0.2019 \\
\hline \%Energy from fat & $15.4 \pm 5.0$ & $15.9 \pm 5.2$ & 0.1322 & $13.0 \pm 5.2$ & $14.0 \pm 5.4$ & 0.0002 \\
\hline $\begin{array}{l}\text { \%Energy from } \\
\text { carbohydrate }\end{array}$ & $69.5 \pm 6.5$ & $71.0 \pm 6.9$ & $<0.0001$ & $72.4 \pm 6.6$ & $73.4 \pm 7.5$ & 0.0062 \\
\hline
\end{tabular}

${ }^{1}$ Data are expressed as mean \pm standard deviation. ${ }^{2}$ Student's $t$-test was used to analyze $p$-value.

\subsection{Metabolic Parameters According to the Percentage of Energy from Total Sugar Intake}

Men who received $>20 \%$ TEI from total sugar had higher WC $(p=0.0481)$ and lower HDL-cholesterol $(p=0.0067)$ than those who derived $\leq 20 \%$ TEI from total sugar (Table 3$)$. This trend was also evident in women $(p=0.0455)$. 
Table 3. Metabolic parameters of participants according to the percentage of energy from total sugar intake ${ }^{1}$.

\begin{tabular}{|c|c|c|c|c|c|c|}
\hline \multirow[b]{2}{*}{ Metabolic Parameter } & \multicolumn{3}{|c|}{ Men $(n=3751)$} & \multicolumn{3}{|c|}{ Women $(n=3254)$} \\
\hline & $\begin{array}{c}\% \text { Energy from } \\
\text { Total Sugar } \leq 20 \\
\quad(n=3404)\end{array}$ & $\begin{array}{c}\text { \%Energy from } \\
\text { Total Sugar }>20 \\
\quad(n=347)\end{array}$ & $p$-Value ${ }^{2}$ & $\begin{array}{c}\text { \%Energy from } \\
\text { Total Sugar } \leq 20 \\
\quad(n=2734)\end{array}$ & $\begin{array}{l}\text { \%Energy from } \\
\text { Total Sugar }>20 \\
\quad(n=520)\end{array}$ & $p$-Value ${ }^{2}$ \\
\hline $\mathrm{WC}(\mathrm{cm})$ & $83.4 \pm 7.5$ & $84.7 \pm 7.9$ & 0.0481 & $81.6 \pm 9.5$ & $83.4 \pm 9.5$ & 0.0455 \\
\hline SBP (mmHg) & $124.6 \pm 17.4$ & $125.3 \pm 17.4$ & 0.6158 & $124.0 \pm 19.9$ & $126.0 \pm 19.4$ & 0.3130 \\
\hline $\mathrm{DBP}(\mathrm{mmHg})$ & $83.2 \pm 11.3$ & $83.1 \pm 11.2$ & 0.5797 & $80.3 \pm 12.0$ & $81.1 \pm 11.5$ & 0.9307 \\
\hline FBS (mg/dL) & $90.7 \pm 23.9$ & $87.6 \pm 21.2$ & 0.1702 & $85.3 \pm 18.8$ & $84.6 \pm 19.9$ & 0.5972 \\
\hline No. of MS components & $1.7 \pm 1.2$ & $1.8 \pm 1.2$ & 0.0630 & $1.9 \pm 1.3$ & $2.0 \pm 1.3$ & 0.8220 \\
\hline Insulin $(\mu \mathrm{IU} / \mathrm{mL})$ & $7.0 \pm 4.1$ & $7.2 \pm 4.5$ & 0.9684 & $8.0 \pm 5.2$ & $8.4 \pm 4.6$ & 0.8490 \\
\hline HbA1c $(\%)$ & $5.8 \pm 0.9$ & $5.8 \pm 0.9$ & 0.5023 & $5.7 \pm 0.8$ & $5.8 \pm 0.8$ & 0.2990 \\
\hline HOMA-IR & $1.6 \pm 1.1$ & $1.6 \pm 1.1$ & 0.6196 & $1.7 \pm 1.2$ & $1.8 \pm 1.1$ & 0.9646 \\
\hline $\mathrm{CRP}(\mathrm{mg} / \mathrm{dL})$ & $0.2 \pm 0.5$ & $0.2 \pm 0.4$ & 0.7928 & $0.2 \pm 0.4$ & $0.2 \pm 0.5$ & 0.2783 \\
\hline LDL-cholesterol (mg/dL) & $113.9 \pm 35.7$ & $109.7 \pm 31.4$ & 0.9834 & $117.0 \pm 32.6$ & $114.2 \pm 29.9$ & 0.2525 \\
\hline
\end{tabular}

${ }^{1}$ Data are expressed as mean \pm standard deviation. ${ }^{2}$ General linear model (GLM) was used to analyze $p$-value after adjusting for age (years), energy intake (kcal), residential location (Ansung and Ansan), educational level (elementary school, middle school/high school, college/graduate school), alcohol consumption (non-drinker, ex-drinker, current drinker), smoking behavior (non-smoker, ex-smoker, current smoker), physical activity (in days; none or $\geq 30 \mathrm{~min} /$ day), and menopausal status ("yes" or "no"; only women). WC, waist circumference; SBP, systolic blood pressure; DBP, diastolic blood pressure; FBS, fasting blood glucose; MS, metabolic syndrome; HbA1c, glycosylated hemoglobin; HOMA-IR, homeostasis model assessment estimate of insulin resistance; CRP, c-reactive protein; $\mathrm{AI}$, atherogenic index.

\subsection{Association between the Percentage of Energy from Total Sugar Intake and Cardiometabolic Biomarkers Risk}

Men with $>20 \%$ TEI from total sugar had a higher odds of obesity (defined as BMI $\geq 25 \mathrm{~kg} / \mathrm{m}^{2}$ ) $(\mathrm{OR}=1.491,95 \% \mathrm{CI}=1.162-1.914)$ after adjusting for covariates (Table 4$)$ in comparison to the men with $\leq 20 \%$ TEI from total sugar Except for obesity, there was no difference in the odds of other cardiometabolic biomarker risk factors between the two groups of men. There was no difference in the odds of all cardiometabolic biomarker risk factors between the two groups of women. 
Table 4. Multivariate odds ratios (OR) and 95\% confidence intervals (CI) of cardiometabolic biomarkers risk according to the percentage of energy from total sugar intake ${ }^{1}$.

\begin{tabular}{|c|c|c|c|c|}
\hline \multirow[b]{2}{*}{ Cardiometabolic Biomarker } & \multicolumn{2}{|c|}{ Men $(n=3751)$} & \multicolumn{2}{|c|}{ Women $(n=3254)$} \\
\hline & $\begin{array}{c}\text { \%Energy from } \\
\text { Total Sugar } \leq 20 \\
(n=3404)\end{array}$ & $\begin{array}{c}\text { \%Energy from } \\
\text { Total Sugar }>20 \\
\quad(n=347)\end{array}$ & $\begin{array}{l}\text { \%Energy from } \\
\text { Total Sugar } \leq 20 \\
\quad(n=2734)\end{array}$ & $\begin{array}{c}\% \text { Energy from } \\
\text { Total Sugar }>20 \\
(n=520)\end{array}$ \\
\hline Obesity $^{2}$ & & & & \\
\hline No. of case (\%) & 1149 (33.8) & $128(36.9)$ & $971(35.5)$ & $188(36.2)$ \\
\hline $\begin{array}{c}\text { Multivariate OR }(95 \% \mathrm{CI}) \\
\text { High insulin } 3\end{array}$ & 1.000 (ref) & $1.491(1.162-1.914)$ & 1.000 (ref) & $1.143(0.932-1.401)$ \\
\hline No. of case (\%) & $822(24.2)$ & $82(23.6)$ & $903(33.0)$ & $204(39.2)$ \\
\hline $\begin{array}{c}\text { Multivariate OR }(95 \% \mathrm{CI}) \\
\text { High } \mathrm{HbA} 1 \mathrm{c}^{4}\end{array}$ & 1.000 (ref) & $0.945(0.723-1.237)$ & 1.000 (ref) & $1.162(0.953-1.417)$ \\
\hline No. of case (\%) & $298(8.8)$ & $27(7.8)$ & $228(8.3)$ & $40(7.7)$ \\
\hline $\begin{array}{c}\text { Multivariate OR }(95 \% \text { CI }) \\
\text { High HOMA-IR }\end{array}$ & 1.000 (ref) & $0.906(0.592-1.385)$ & 1.000 (ref) & $0.898(0.626-1.287)$ \\
\hline No. of case (\%) & $842(24.7)$ & $80(23.1)$ & $740(27.1)$ & $159(30.6)$ \\
\hline $\begin{array}{c}\text { Multivariate OR }(95 \% \mathrm{CI}) \\
\text { High CRP } 6\end{array}$ & 1.000 (ref) & $0.919(0.701-1.204)$ & 1.000 (ref) & $1.075(0.871-1.327)$ \\
\hline No. of case (\%) & $92(2.7)$ & $11(3.2)$ & $54(2.0)$ & $12(2.3)$ \\
\hline $\begin{array}{c}\text { Multivariate OR }(95 \% \mathrm{CI}) \\
\text { High LDL-cholesterol }^{7}\end{array}$ & 1.000 (ref) & $1.263(0.654-2.436)$ & 1.000 (ref) & $1.127(0.590-2.152)$ \\
\hline No. of case (\%) & $1100(32.3)$ & $91(26.2)$ & 885 (32.4) & $147(28.3)$ \\
\hline $\begin{array}{c}\text { Multivariate OR }(95 \% \mathrm{CI}) \\
\text { High AI }^{8}\end{array}$ & 1.000 (ref) & $0.908(0.697-1.182)$ & 1.000 (ref) & $0.850(0.685-1.054)$ \\
\hline No. of case (\%) & $371(10.9)$ & $36(10.4)$ & $211(7.7)$ & $35(6.7)$ \\
\hline Multivariate OR (95\% CI) & 1.000 (ref) & $0.999(0.684-1.457)$ & 1.000 (ref) & $0.879(0.601-1.287)$ \\
\hline
\end{tabular}

${ }^{1}$ Multiple logistic regression was used to estimate OR (95\% CI) after adjusting for age (years), energy intake (kcal), residential location (Ansung and Ansan), educational level (elementary school, middle school/high school, college/graduate school), alcohol consumption (non-drinker, ex-drinker, current drinker), smoking behavior (non-smoker, ex-smoker, current smoker), physical activity (in days; none or $\geq 30 \mathrm{~min} /$ day), and menopausal status ("yes" or "no"; only women). ${ }^{2}$ Obesity (BMI $\left.\geq 25 \mathrm{~kg} / \mathrm{m}^{2}\right) .{ }^{3} \mathrm{High}$ insulin $(\geq 9 \mu \mathrm{IU} / \mathrm{mL}) .{ }^{4} \mathrm{High} \mathrm{HbA1c}(\geq 6.5 \%) .{ }^{5} \mathrm{high}$ HOMA-IR ([fasting glucose $\times$ fasting insulin] $/ 405 ;>2) .{ }^{6}$ High CRP $(>1 \mathrm{mg} / \mathrm{dL}) .{ }^{7}$ High LDL-cholesterol $(\geq 130 \mathrm{mg} / \mathrm{dL})$. ${ }^{8}$ High AI ([total cholesterol-HDL-cholesterol]/HDL-cholesterol; >5). HbA1c, glycosylated hemoglobin; HOMA-IR, homeostasis model assessment estimate of insulin resistance; CRP, c-reactive protein; AI, atherogenic index.

\subsection{Association between the Percentage of Energy from Total Sugar Intake and Metabolic Syndrome}

Table 5 shows that men who derived $>20 \%$ TEI from total sugar had a higher odds of low HDL-cholesterol $(\mathrm{OR}=1.313,95 \% \mathrm{CI}=1.038-1.660)$ and metabolic syndrome $(\mathrm{OR}=1.332,95 \%$ $\mathrm{CI}=1.038-1.709)$ when compared with those who received $\leq 20 \%$ TEI from total sugar There was no difference in odds of metabolic syndrome between the two groups of women. 
Table 5. Multivariate odds ratios (OR) and 95\% confidence intervals (CI) of metabolic syndrome according to the percentage of energy from total sugar intake ${ }^{1}$.

\begin{tabular}{|c|c|c|c|c|}
\hline \multirow[b]{2}{*}{$\begin{array}{l}\text { Metabolic Syndrome } \\
\text { Parameter }\end{array}$} & \multicolumn{2}{|c|}{ Men $(n=3751)$} & \multicolumn{2}{|c|}{ Women $(n=3254)$} \\
\hline & $\begin{array}{c}\text { \%Energy from } \\
\text { Total Sugar } \leq 20 \\
(n=3404)\end{array}$ & $\begin{array}{c}\text { \%Energy from } \\
\text { Total Sugar >20 } \\
(n=347)\end{array}$ & $\begin{array}{c}\text { \%Energy from } \\
\text { Total Sugar } \leq 20 \\
(n=2734)\end{array}$ & $\begin{array}{c}\text { \%Energy from } \\
\text { Total Sugar }>20 \\
\quad(n=520)\end{array}$ \\
\hline \multicolumn{5}{|l|}{ Abdominal obesity ${ }^{2}$} \\
\hline No. of case $(\%)$ & $606(17.8)$ & $81(23.3)$ & $948(34.7)$ & $213(41.0)$ \\
\hline Multivariate OR $(95 \% \mathrm{CI})$ & 1.000 (ref) & $1.218(0.926-1.604)$ & 1.000 (ref) & $1.129(0.917-1.389)$ \\
\hline \multicolumn{5}{|l|}{ High blood pressure ${ }^{3}$} \\
\hline No. of case $(\%)$ & 1629 (47.9) & $185(53.3)$ & 1165 (42.6) & $237(45.6)$ \\
\hline Multivariate OR $(95 \% \mathrm{CI})$ & 1.000 (ref) & $1.194(0.945-1.509)$ & 1.000 (ref) & $1.011(0.822-1.245)$ \\
\hline \multicolumn{5}{|l|}{ High glucose ${ }^{4}$} \\
\hline No. of case $(\%)$ & 535 (15.7) & 35 (10.1) & 235 (8.6) & $33(6.4)$ \\
\hline Multivariate OR $(95 \% \mathrm{CI})$ & 1.000 (ref) & $0.716(0.494-1.038)$ & 1.000 (ref) & $0.755(0.513-1.109)$ \\
\hline \multicolumn{5}{|l|}{ High triglycerides ${ }^{5}$} \\
\hline No. of case $(\%)$ & $1641(48.2)$ & $174(50.1)$ & $959(35.1)$ & $187(36.0)$ \\
\hline Multivariate OR $(95 \% \mathrm{CI})$ & 1.000 (ref) & $1.121(0.892-1.410)$ & 1.000 (ref) & $1.041(0.848-1.277)$ \\
\hline \multicolumn{5}{|l|}{ Low HDL-cholesterol ${ }^{6}$} \\
\hline No. of case $(\%)$ & $1218(35.8)$ & $150(43.2)$ & $1930(70.6)$ & $367(70.6)$ \\
\hline Multivariate OR $(95 \% \mathrm{CI})$ & 1.000 (ref) & $1.313(1.038-1.660)$ & 1.000 (ref) & $0.979(0.792-1.210)$ \\
\hline \multicolumn{5}{|l|}{ Metabolic syndrome ${ }^{7}$} \\
\hline No. of case (\%) & $823(24.2)$ & $107(30.8)$ & $900(32.9)$ & $174(33.5)$ \\
\hline Multivariate OR $(95 \% \mathrm{CI})$ & 1.000 (ref) & $1.332(1.038-1.709)$ & 1.000 (ref) & $0.922(0.743-1.143)$ \\
\hline \multicolumn{5}{|c|}{$\begin{array}{l}\left.{ }^{1} \text { Analyzed by logistic regression for OR ( } 95 \% \mathrm{CI} \text { ) after adjusting for age (in years; } 40-69\right) \text {, energy intake, residential } \\
\text { location (Ansung and Ansan), educational level (elementary school, middle school/high school, college/graduate } \\
\text { school), alcohol consumption (non-drinker, ex-drinker, current drinker), smoking behavior (non-smoker, ex-smoker, } \\
\text { current smoker), physical activity (in days; none or } \geq 30 \mathrm{~min} / \mathrm{day} \text { ), and menopausal status ("yes" or "no"; only } \\
\text { women). }{ }^{2} \text { Abdominal obesity (WC: male }>90 \mathrm{~cm} \text {, female }>85 \mathrm{~cm} \text { ). }{ }^{3} \text { High blood pressure (SBP } \geq 130 \mathrm{mmHg} \\
\left.\left.\text { or DBP } \geq 85 \mathrm{mmHg}){ }^{4} \text { High glucose (FBS } \geq 100 \mathrm{mg} / \mathrm{dL}\right) .{ }^{5} \mathrm{High} \text { triglycerides (triglycerides } \geq 150 \mathrm{mg} / \mathrm{dL}\right) .{ }^{6} \mathrm{Low} \\
\mathrm{HDL} \text {-cholesterol (HDL-cholesterol: male }<40 \mathrm{mg} / \mathrm{dL} \text {, female }<50 \mathrm{mg} / \mathrm{dL}) .{ }^{7} \text { Metabolic syndrome (WC, triglycerides, } \\
\text { blood pressure, FBS, HDL-cholesterol; } \geq 3 \text { of } 5 \text { ). WC, waist circumference; SBP, systolic blood pressure; DBP, diastolic } \\
\text { blood pressure; FBS, fasting blood glucose. }\end{array}$} \\
\hline
\end{tabular}

\section{Discussion}

This study found that deriving $>20 \%$ TEI from total sugar was associated with higher odds of obesity, low HDL-cholesterol, and metabolic syndrome among Korean men aged 40-69 years, but not among women in the same age range.

The study of the relationship between sugar intake and chronic diseases has been mainly focused on SSB, with insufficient analysis of total sugar intake. In addition, investigations into the association between total sugar intake and chronic diseases, including metabolic syndrome, in adults who are not Korean adolescents are even rarer. Recently, the KFDA reported the results of a study examining the relationship between the total sugar intake (calculated using two-day 24-h recall data) and chronic diseases (using the KoGES data). It found that the risk of obesity, diabetes, and hypertension was not affected by whether total sugar intake was above or below $20 \%$ TEI. However, the risk of overweight was higher in men who derived $>20 \%$ TEI from total sugar compared with those who received $<20 \%$ TEI from total sugar [26].

The proportion of processed foods (56.8\%) contributing to total sugar intake of Koreans was higher than that from food in which sugars occur naturally, and the top five processed food sources of total sugar intake were granulated sugar, carbonated beverages, coffee, bread, and fruit and vegetable drinks [11]. In other words, among processed foods, SSB were found to be the main source of sugar intake. It has been reported that SSB negatively affect health because they generally do not give a feeling of fullness and only increase energy density without providing other nutrients. Shin et al. [27] argued that high consumption of SSB was closely linked to a higher prevalence of obesity and metabolic syndrome in the Korean population. Analysis by Kwak et al. [28] demonstrated that among 5575 middle-aged participants in the KoGES, those in the highest SSB consumption group had a $21 \%$ greater risk of hypertension than those in the lowest SSB consumption group, and this association was 
most relevant for the participants with obesity. This relationship between SSB and chronic diseases has also been reported in Asian [29,30] and Western [31,32] populations.

There are several possible mechanisms explaining the link between SSB intake and chronic disease. First, the energy consumed in the form of SSB is rapidly absorbed, resulting in a positive energy balance, which, in turn, leads to increased weight since more energy is consumed in the next meal [33]. Second, apart from the weight gain effect, SSB intake increases the dietary glycemic load and fructose intake, which increases insulin resistance, beta-cell function, inflammation, hypertension, accumulation of visceral fat, and, eventually, increases the risk of metabolic syndrome, type 2 diabetes, and cardiovascular disease [34]. Moreover, artificial sweeteners have also been reported to enhance the risk of glucose intolerance. Continued consumption of artificial sweeteners can alter the composition and function of the gut microbiota, which may lead to the induction of metabolic abnormalities, such as glucose intolerance [35].

Interestingly, our study found a positive association between total sugar intake and metabolic syndrome in men, but not in women, even though the proportion of the highest consumption group in men was lower than that in women. It is unclear why only men showed a positive association between total sugar intake and metabolic disease. Different outcomes in the association of total sugar or SSB with metabolic disease between sexes can be found in the literature. In a cross-sectional study, SSB intake was significantly associated with obesity in men, but metabolic syndrome and obesity in women [27]. In other work, a positive association between total sugar intake and overweight was exclusively observed in men [26]. When examining the data of 5797 middle-aged Korean men and women, Kang and Kim [36] revealed that frequent soft drink consumption ( $>4$ servings/week) was associated with an increased risk of developing metabolic syndrome and its components only in women. Elsewhere, women, but not men, who consumed $\geq 1 \mathrm{SSB} /$ day had an increased incidence of type 2 diabetes [30]. These sex-dependent results might be explained by dietary intake and lifestyle differences between men and women that could alter hormone levels and metabolic profile, which could affect obesity or metabolic disease.

In the present study, in men, but not women, there was a significant association between high sugar intake and unhealthy lifestyle (such as smoking). High sugar consumption (particularly as SSB) is a known marker of an unhealthy lifestyle. Long-term high intake of soft drinks ( $\geq 3$ times per week) has been significantly associated with a lower level of physical activity and a greater degree of smoking than long-term, low intake of soft drinks ( $<3$ times per week) [37]. Another study found that high soft drink intake ( $\geq$ twice a day) was associated with more smoking than low soft drink intake $(<$ once a day) [32].

Low estrogen concentration causes low HDL-cholesterol and leads to resistance to insulin-mediated glucose [38,39] because estrogen protects HDL-cholesterol by suppressing hepatic lipase and is tightly associated with lipoprotein profiles $[40,41]$. Since these processes contribute to obesity and type 2 diabetes, they increase the risk of exposure to metabolic syndrome and cardiovascular disease risk factors [33,42]. Therefore, women maintaining an HDL-cholesterol level at $10 \mathrm{mg} / \mathrm{dL}$ higher than men by estrogen have a relatively lower risk of metabolic syndrome and cardiovascular disease [43]. As a result, differences by sex can be interpreted according to evidence that estrogen's effect on lipid metabolism and lifestyle may contribute to the association between sugar intake and metabolic syndrome.

In our study, participants who exceeded $20 \%$ TEI from total sugar had higher nutrient intake than those with $\leq 20 \%$ TEI from total sugar. These results may be caused by differences in the major sources of sugar, highlighting the importance of measuring sugar intake, considering the main dietary source of sugar. Physiological metabolic pathways are affected by the form of sugars. Unlike simple sugars that are absorbed in the upper half of the gastrointestinal tract, complex carbohydrates are absorbed after decomposition by microbes in the large intestine [44]. During this process, short-chain fatty acids are produced, stimulating enteroendocrine pathways, thereby increasing the levels of satiety hormones [44]. In comparing solid intake with liquid intake, the masticatory movement of eating solid food triggers satiety hormones, which can reduce total energy intake [45]. Furthermore, pancreatic 
exocrine and endocrine mechanisms that act to release insulin function better, and this can enhance the regulation of glucose tolerance [45]. For these reasons, even with isoenergetic sugar intake, the outcomes on health appear different depending on the original form of the food.

This study had several limitations. First, because our data are based on a cross-sectional study, it is difficult to explain the causal relationship between sugar intake and metabolic syndrome. Therefore, a further prospective cohort study is needed. Second, it is difficult to generalize the results to the entire Korean population because we only selected participants in Ansan and Ansung. Therefore, the results should be interpreted carefully. Although the study had some limitations, we analyzed the data after controlling for potential confounders to reduce the impact of these limitations. To the best of our knowledge, this is the first study to examine the associations of total sugar intake with obesity and metabolic syndrome (including its discrete components) at the same time, among middle-aged Korean adults. In particular, it is important to examine total sugar intake as a contribution to energy rather than total sugar intake in relation to obesity or metabolic syndrome.

Unlike the ongoing research activities in the West, research on sugar intake and chronic diseases in Korea is at the beginning stage. Korea and the West have different diets, and so it is reasonable to propose that there will be differences in their vulnerability to chronic diseases in relation to their diet. This study examined the relevance of total sugar intake as a risk factor of metabolic syndrome in middle-aged Korean adults. It sets a precedent for further research in this population that will confirm or not the suggestive evidence provided in this investigation that found total sugar intake is significantly associated with metabolic syndrome in middle-aged Korean men (40-69 years) but not women. Furthermore, the results of this study can be used as a scientific basis for setting the target level in order to gradually reduce not only the sugar intake of Koreans but populations worldwide. The data may also be used to suggest the appropriate policy for reducing the sugar intake of middle-aged adults.

\section{Conclusions}

In conclusion, this study found that total sugar intake was significantly associated with metabolic syndrome in middle-aged Korean men (40-69 years). Total sugar intake exceeding $>20 \%$ of the TEI was associated with higher risks of low HDL-cholesterol, obesity (defined as BMI $\geq 25 \mathrm{~kg} / \mathrm{m}^{2}$ ), and metabolic syndrome in men. While total sugar intake is important in terms of its contribution to the ratio of energy intake, other added-sugar/free-sugar intake levels and food sources are important and their causal relationship with metabolic syndrome should be identified using this comprehensive sugar intake evaluation.

Author Contributions: Conceptualization and design, O.K. and H.K.; statistical analysis, E.H.S. and H.K.; writing, E.H.S. and H.K.; critical review, O.K.

Funding: This research was funded by the Bio-synergy Research Project grant number [NRF2012M3A9C4048761] of the Ministry of Science, ICT and Future Planning through the National Research Foundation and RP-Grant 2018 of Ewha Womans University, South Korea.

Conflicts of Interest: The authors declare no conflict of interest.

\section{References}

1. WHO. Diet, Nutrition and the Prevention of Chronic Diseases; Technical Report Series 916; Report of a Joint WHO/FAO Expert Consultation; 28 January-1 February 2002; World Health Organization: Geneva, Switzerland, 2003.

2. Brand-Miller, J.C.; Barclay, A.W. Declining consumption of added sugars and sugar-sweetened beverages in Australia: A challenge for obesity prevention. Am. J. Clin. Nutr. 2017, 105, 854-863. [CrossRef] [PubMed]

3. Erickson, J.; Slavin, J. Total, added, and free sugars: Are restrictive guidelines science-based or achievable? Nutrients 2015, 7, 2866-2878. [CrossRef] [PubMed]

4. Gibson, S.; Francis, L.; Newens, K.; Livingstone, B. Associations between free sugars and nutrient intakes among children and adolescents in the UK. Br. J. Nutr. 2016, 116, 1265-1274. [CrossRef] [PubMed] 
5. Malik, V.S.; Hu, F.B. Sweeteners and risk of obesity and type 2 diabetes: The role of sugar-sweetened beverages. Curr. diabetes Rep. 2012, 12, 195-203. [CrossRef] [PubMed]

6. Rippe, J.M.; Angelopoulos, T.J. Relationship between added sugars consumption and chronic disease risk factors: Current understanding. Nutrients 2016, 8, 697. [CrossRef]

7. World Health Organization. Guideline: Sugars Intake for Adults and Children; World Health Organization: Geneva, Switzerland, 2015.

8. U.S. Department of Agriculture; U.S. Department of Health and Human Services. Dietary Guidelines for Americans 2010, 7th ed.; U.S. Government Printing Office: Washington, DC, USA, 2010.

9. Food Standards Agency. FSA Nutrient and Food Based Guidelines for UK Institutions; Food Standards Agency: London, UK, 2006.

10. Ministry of Health and Welfare, The Korean Nutrition Society. Dietary Reference Intakes for Koreans 2015; The Korean Nutrition Society: Seoul, Korea, 2015.

11. Lee, H.S.; Kwon, S.O.; Yon, M.Y.; Kim, D.; Lee, J.Y.; Nam, J.; Park, S.J.; Yeon, J.Y.; Lee, S.K.; Lee, H.Y.; et al. Dietary total sugar intake of Koreans: Based on the Korea National Health and Nutrition Examination Survey (KNHANES), 2008-2011. J. Nutr. Health 2014, 47, 268-276. [CrossRef]

12. Kang, J.H. The Correlation Analysis of Sugars Excess-Intake and Obesity or Chronic Disease and the Development of Sugar-Reduction Model; Korea Food and Drug Administration: Cheongwon, Korea, 2013.

13. Newens, K.J.; Walton, J. A review of sugar consumption from nationally representative dietary surveys across the world. J. Hum. Nutr. Diet. 2016, 29, 225-240. [CrossRef]

14. Oh, K.S. A Study on the Dietary Pattern and Intake of Potentially Hazardous Nutrients among Korean Adults; Korea Food and Drug Administration: Cheongju, Korea, 2017.

15. Kim, S. Changes in Food Consumption in Korea. Available online: Ap.fftc.agnet.org/ap_db.php?id=940 (accessed on 14 November 2018).

16. Kwon, Y.S.; Ju, S.Y. Trends in nutrient intakes and consumption while eating-out among Korean adults based on Korea National Health and Nutrition Examination Survey (1998-2012) data. Nutr. Res. Pract. 2014, 8, 670-678. [CrossRef]

17. Lim, S.; Shin, H.; Song, J.H.; Kwak, S.H.; Kang, S.M.; Won, Y.J.; Choi, S.H.; Cho, S.I.; Park, K.S.; Lee, H.K.; et al. Increasing prevalence of metabolic syndrome in Korea: The Korean National Health and Nutrition Examination Survey for 1998-2007. Diabetes Care 2011, 34, 1323-1328. [CrossRef]

18. Kim, H.R.; Han, M.A. Association between serum liver enzymes and metabolic syndrome in Korean adults. Int. J. Environ. Res. Public Health 2018, 15, 1658. [CrossRef]

19. Yang, Y.M.; Shin, B.C.; Son, C.; Ha, I.H. An analysis of the associations between gender and metabolic syndrome components in Korean adults: A national cross-sectional study. BMC Endocr. Disord. 2019, $19,67$. [CrossRef] [PubMed]

20. Lim, S.; Jang, H.C.; Lee, H.K.; Kimm, K.C.; Park, C.; Cho, N.H. A rural-urban comparison of the characteristics of the metabolic syndrome by gender in Korea: The Korean Health and Genome Study (KHGS). J. Endocrinol. Investig. 2006, 29, 313-319. [CrossRef] [PubMed]

21. Ray, S.; Bairagi, A.K.; Guha, S.; Ganguly, S.; Ray, D.; Basu, A.K.; Sinha, A. A simple way to identify insulin resistance in non-diabetic acute coronary syndrome patients with impaired fasting glucose. Indian J. Endocrinol. Metab. 2012, 16, S460-S464. [CrossRef]

22. Nam, S.M.; Ha, E.H.; Suh, Y.J.; Park, H.; Chang, M.H.; Seo, J.H.; Kim, B.M. Effect of obesity and blood lipid profiles on hyperlipidemia in adults aged over 40 years. Korean J. Obes. 2008, 17, 20-28.

23. Grundy, S.M.; Brewer, H.B., Jr.; Cleeman, J.I.; Smith, S.C., Jr.; Lenfant, C. Definition of metabolic syndrome: Report of the National Heart, Lung, and Blood Institute/American Heart Association conference on scientific issues related to definition. Circulation 2004, 109, 433-438. [CrossRef] [PubMed]

24. Lee, S.; Park, H.S.; Kim, S.M.; Kwon, H.S.; Kim, D.Y.; Kim, D.J.; Cho, G.J.; Han, J.H.; Kim, S.R.; Park, C.Y.; et al. Cut-off points of waist circumference for defining abdominal obesity in the Korean population. Korean J. Obes. 2006, 15, 1-9.

25. Lee, H.S.; Kim, C.I.; Kim, D.; Yon, M.; Lee, J.Y.; Nam, J.; Park, S.J.; Ahn, J.H.; Hwang, E.J.; Moon, J.H. Sugar Database Compilation for Commonly Consumed Foods; Korea Health Industry Development Institute: Cheongju, Korea, 2015.

26. Oh, K.S. A Study on the Relation of Nutrient Intake and Disease among Korean Adults; Korea Food and Drug Administration: Cheongju, Korea, 2018. 
27. Shin, S.; Kim, S.A.; Ha, J.; Lim, K. Sugar-sweetened beverage consumption in relation to obesity and metabolic syndrome among Korean adults: A cross-sectional study from the 2012-2016 Korean National Health and Nutrition Examination Survey (KNHANES). Nutrients 2018, 10, 1467. [CrossRef]

28. Kwak, J.H.; Jo, G.; Chung, H.K.; Shin, M.J. Association between sugar-sweetened beverage consumption and incident hypertension in Korean adults: A prospective study. Eur. J. Nutr. 2019, 58, 1009-1017. [CrossRef]

29. Odegaard, A.O.; Koh, W.P.; Arakawa, K.; Yu, M.C.; Pereira, M.A. Soft drink and juice consumption and risk of physician-diagnosed incident type 2 diabetes: The Singapore Chinese Health Study. Am. J. Epidemiol. 2010, 171, 701-708. [CrossRef]

30. Papier, K.; D'Este, C.; Bain, C.; Banwell, C.; Seubsman, S.; Sleigh, A.; Jordan, S. Consumption of sugar-sweetened beverages and type 2 diabetes incidence in Thai adults: Results from an 8-year prospective study. Nutr. Diabetes 2017, 7, e283. [CrossRef]

31. Malik, V.S.; Popkin, B.M.; Bray, G.A.; Després, J.P.; Willett, W.C.; Hu, F.B. Sugar-sweetened beverages and risk of metabolic syndrome and type 2 diabetes: A meta-analysis. Diabetes Care 2010, 33, 2477-2483. [CrossRef] [PubMed]

32. Dhingra, R.; Sullivan, L.; Jacques, P.F.; Wang, T.J.; Fox, C.S.; Meigs, J.B.; D'Agostino, R.B.; Gaziano, J.M.; Vasan, R.S. Soft drink consumption and risk of developing cardiometabolic risk factors and the metabolic syndrome in middle-aged adults in the community. Circulation 2007, 116, 480-488. [CrossRef] [PubMed]

33. Malik, V.S.; Popkin, B.M.; Bray, G.A.; Després, J.; Hu, F.B. Sugar-sweetened beverages, obesity, type 2 diabetes mellitus, and cardiovascular disease risk. Circulation 2010, 121, 1356-1364. [CrossRef] [PubMed]

34. Bray, G.A. Fructose and risk of cardiometabolic disease. Curr. Atheroscler. Rep. 2012, 14, 570-578. [CrossRef] [PubMed]

35. Suez, J.; Korem, T.; Zeevi, D.; Zilberman-Schapira, G.; Thaiss, C.A.; Maza, O.; Israeli, D.; Zmora, N.; Gilad, S.; Weinberger, A.; et al. Artificial sweeteners induce glucose intolerance by altering the gut microbiota. Nature 2014, 514, 181-186. [CrossRef] [PubMed]

36. Kang, Y.; Kim, J. Soft drink consumption is associated with increased incidence of the metabolic syndrome only in women. Br. J. Nutr. 2017, 117, 315-324. [CrossRef] [PubMed]

37. Kvaavik, E.; Andersen, L.F.; Klepp, K.I. The stability of soft drinks intake from adolescence to adult age and the association between long-term consumption of soft drinks and lifestyle factors and body weight. Public Health Nutr. 2005, 8, 149-157. [CrossRef] [PubMed]

38. Walsh, B.W.; Schiff, I.; Rosner, B.; Greenberg, L.; Ravnikar, V.; Sacks, F.M. Effects of postmenopausal estrogen replacement on the concentrations and metabolism of plasma lipoproteins. N. Engl. J. Med. 1991, 325, 1196-1204. [CrossRef]

39. Jeppesen, J.; Facchini, F.S.; Reaven, G.M. Individuals with high total cholesterol/HDL cholesterol ratios are insulin resistant. J. Intern. Med. 1998, 243, 293-298. [CrossRef]

40. Perret, B.; Mabile, L.; Martinez, L.; Tercé, F.; Barbaras, R.; Collet, X. Hepatic lipase: Structure/function relationship, synthesis, and regulation. J. Lipid Res. 2002, 43, 1163-1169. [CrossRef]

41. Knowlton, A.A.; Lee, A.R. Estrogen and the cardiovascular system. Pharmacol. Ther. 2012, 135, 54-70. [CrossRef] [PubMed]

42. Pennings, N.; Jaber, J.; Ahiawodzi, P. Ten-year weight gain is associated with elevated fasting insulin levels and precedes glucose elevation. Diabetes Metab. Res. Rev. 2018, 34, e2986. [CrossRef] [PubMed]

43. Bittner, V. Perspectives on dyslipidemia and coronary heart disease in women. J. Am. Coll. Cardiol. 2005, 46, 1628-1635. [CrossRef] [PubMed]

44. Freeman, C.R.; Zehra, A.; Ramirez, V.; Wiers, C.E.; Volkow, N.D.; Wang, G.J. Impact of sugar on the body, brain, and behavior. Front. Biosci. (Landmark Ed.) 2018, 23, 2255-2266. [CrossRef] [PubMed]

45. DiMeglio, D.P.; Mattes, R.D. Liquid versus solid carbohydrate: Effects on food intake and body weight. Int. J. Obes. Relat. Metab. Disord. 2000, 24, 794-800. [CrossRef] [PubMed]

(C) 2019 by the authors. Licensee MDPI, Basel, Switzerland. This article is an open access article distributed under the terms and conditions of the Creative Commons Attribution (CC BY) license (http://creativecommons.org/licenses/by/4.0/). 